\title{
Analysis and modeling of proton beam loss and emittance growth in the Relativistic Heavy Ion Collider
}

\author{
Y. Luo, W. Fischer, and S. White \\ Brookhaven National Laboratory, Upton, New York 11973, USA
}

(Received 7 September 2015; published 4 February 2016)

\begin{abstract}
The Relativistic Heavy Ion Collider (RHIC) at Brookhaven National Laboratory has been operating since 2000. Over the past decade, thanks to the continuously increased bunch intensity and reduced $\beta^{*} \mathrm{~s}$ at the interaction points, the peak luminosity in the polarized proton operation has been increased by more than two orders of magnitude. In this article, we will present the operational observations at the routine proton physics stores. The mechanisms for the beam loss, transverse emittance growth, and bunch lengthening are analyzed. Numerical calculations and multiparticle tracking are used to model these observations.
\end{abstract}

DOI: 10.1103/PhysRevAccelBeams.19.021001

\section{INTRODUCTION}

The Relativistic Heavy Ion Collider (RHIC) at Brookhaven National Laboratory consists of two superconducting rings, the "Blue" ring and the "Yellow" ring. The two rings intersect horizontally at 6 symmetric interaction points (IPs) along a $3.8 \mathrm{~km}$ circumference. During the physics stores, the two beams collide head on at IP6 (STAR detector) and IP8 (PHENIX detector). At other 4 noncollisional IPs, the two beams are always separated vertically. Figure 1 shows the layout of RHIC.

RHIC is capable of colliding heavy ions and polarized protons. In the proton operation, the Figure Of Merit (FOM) is $L P^{2}$ for the single spin program and $L P^{4}$ for the double spin program, where $L$ is the luminosity and $P$ the polarization of proton beams. In order to maximize the FOM, both instantaneous luminosity and polarization as well as their lifetimes have to be maximized.

The luminosity is defined as [1]

$$
L=\frac{N_{p}^{2} N_{c} \gamma f_{\text {rev }}}{4 \pi \epsilon_{n, r m s} \beta^{*}} H\left(\beta^{*}, \sigma_{l}\right) .
$$

Here $N_{p}$ is the proton bunch intensity, $N_{c}$ the number of bunch collisions per turn, $\gamma$ the Lorentz factor, $f_{\text {rev }}$ the revolution frequency, $\epsilon_{n, \mathrm{rms}}$ the rms normalized emittance, $\beta^{*}$ the $\beta$-function at the collisional IPs, and $\sigma_{l}$ the rms bunch length. $H\left(\beta^{*}, \sigma_{l}\right)$ is the luminosity reduction factor due to the hourglass effect.

An increase of luminosity is generally achieved by an increase of the proton bunch intensity and a reduction of the $\beta^{*}$ s at the collisional IPs. Using both methods, the RHIC peak luminosity for protons was increased by more

\footnotetext{
yluo@bnl.gov

Published by the American Physical Society under the terms of the Creative Commons Attribution 3.0 License. Further distribution of this work must maintain attribution to the author(s) and the published article's title, journal citation, and DOI.
}

than two orders of magnitude [2] over the past decade. The peak luminosity in the $2013255 \mathrm{GeV}$ proton run reached $2.45 \times 10^{32} \mathrm{~cm}^{-2} \mathrm{~s}^{-1}$.

However, beam-beam interactions introduce amplitudedependent tune shift and nonlinear resonance driving terms [3]. This proved to be the limiting factor in RHIC for further increase of the bunch intensity and drives a special interest in understanding the underlying mechanisms affecting the beam lifetime, emittance and other relevant parameters for luminosity performance.

In RHIC, there are 111 bunches in each ring. Bunches from different rings collide head on at IP6 and IP8. Most bunches have 2 collisions per turn except 10 bunches from each ring have only 1 collision per turn due to some empty buckets requested by the physics experiments and beam abort. The beam-beam parameter can be used as a gauge for the strength of beam-beam interaction. In the case of one collision, it is defined as [4]

$$
\xi=\frac{N_{p} r_{p}}{4 \pi \epsilon_{n, r m s}}
$$

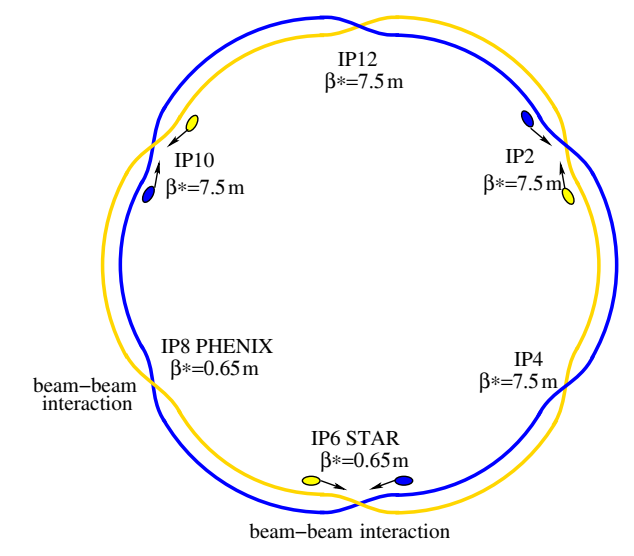

FIG. 1. Layout of RHIC. Particles circulate clockwise in the Blue ring and counterclockwise in the Yellow ring. 
Here $r_{p}$ is the classical radius of proton. As an example, for a bunch intensity $2 \times 10^{11}$ and a normalized emittance $\epsilon_{n, \mathrm{rms}}=2.5 \mu \mathrm{m}$, the beam-beam parameter is $0.01 / \mathrm{IP}$. The higher the bunch intensity is, the larger the beam-beam parameter is. A large beam-beam parameter can lead to beam loss and emittance blowup. The effect of long-range beam-beam interactions in RHIC is negligible $[5,6]$.

The current working point is constrained between $2 / 3$ and $7 / 10$. While $2 / 3$ is a strong third order betatron resonance, $7 / 10$ is a 10th order betatron resonance and more importantly a spin depolarization resonance. To maintain good beam and polarization lifetimes, these two resonances must be avoided during the physics operation. Figure 2 shows the tune footprints with 2 beam-beam interactions. The bunch intensity is $2 \times 10^{11}$, and the lattice tunes are $(28.695,29.685)$ and $(28.685,29.695)$.

As seen on this plot, there is not enough tune space between $2 / 3$ and $7 / 10$ to accommodate the beam-beam tune spread when the proton bunch intensity is larger than $2.0 \times 10^{11}$. The maximum peak bunch intensity we achieved in the $2013255 \mathrm{GeV}$ proton run was $1.85 \times 10^{11}$. Beyond that, a large emittance growth and a bad beam lifetime were observed and therefore was not used in the normal physics operation.

A complete fill in the RHIC proton operation includes injection, energy ramp, rotator ramp, physics store, and ramp-down. To maintain the beam intensity, transverse emittance, and the polarization on the energy and rotator ramps, the orbits, tunes, betatron coupling, and chromaticities have been well controlled [7-10]. Table I lists the machine and beam parameters at store for the 2012 $100 \mathrm{GeV}$ and $2013255 \mathrm{GeV}$ proton runs. The $\beta^{*} \mathrm{~s}$ at the collisional IPs are $0.85 \mathrm{~m}$ and $0.65 \mathrm{~m}$ respectively. In 2012, we also collided protons at $255 \mathrm{GeV}$.

To minimize the detrimental effects from the electron cloud and impedances [11-14], a common 9 MHz rf system

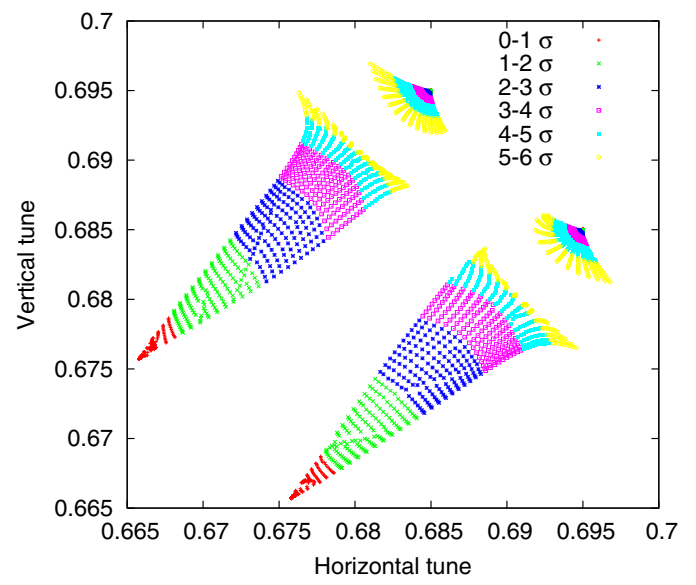

FIG. 2. Tune footprints without and with beam-beam interactions with a bunch intensity $2.0 \times 10^{11}$. Different colors represent particles with different transverse amplitudes.
TABLE I. Beam and optics parameters for the $2012100 \mathrm{GeV}$ and $2013255 \mathrm{GeV}$ RHIC polarized proton runs.

\begin{tabular}{lccc}
\hline \hline & & $100 \mathrm{GeV}$ & $255 \mathrm{GeV}$ \\
\cline { 3 - 4 } Parameters & Unit & in Run-12 & Run-13 \\
\hline Ring circumference & $\mathrm{m}$ & \multicolumn{2}{c}{3833.8451} \\
No. of bunches & & 109 & 111 \\
Bunch intensity, initial & $10^{11}$ & 1.6 & 1.85 \\
Trans. emittance, initial & $\mu \mathrm{m}$ & 3.3 & 3.5 \\
Long. emittance, initial & $\mathrm{eV} \cdot \mathrm{s}$ & 2.0 & 2.0 \\
Working point & $\ldots$ & $(0.695,0.685)$ \\
First order chromaticities & $\ldots$ & \multicolumn{2}{c}{$(1,1)$} \\
$\beta^{*}$ at IP6 and IP8 & $\mathrm{m}$ & 0.85 & 0.65 \\
$\beta$ at IP10/12/2/4 & $\mathrm{m}$ & 7.5 & 7.5 \\
9 MHz rf voltage & $\mathrm{kV}$ & 20 & 20 \\
28 MHz rf voltage & $\mathrm{kV}$ & 360 & 360 \\
197 MHz rf voltage & $\mathrm{kV}$ & 200 & 100 \\
rms bunch length & $\mathrm{cm}$ & 75 & 60 \\
rms momentum spread & $10^{-4}$ & 4.2 & 1.8 \\
Beam-beam parameter/IP & $\ldots$ & 0.007 & 0.007 \\
Peak luminosity & $10^{30} \mathrm{~cm}^{-2} \mathrm{~s}^{-1}$ & 46 & 245 \\
Average/Peak luminosity & $\%$ & 72 & 65 \\
\hline \hline
\end{tabular}

has been commissioned in 2011. The $9 \mathrm{MHz}$ rf cavity provides a large bucket area and reduces the peak bunch current. After reaching the top energy, the bunches are rebucketed from the $9 \mathrm{MHz}$ to $28 \mathrm{MHz}$ rf system. During the physics stores, to produce more collisions in the central area of detectors, $300 \mathrm{kV} 197 \mathrm{MHz}$ rf voltage was added in 2012 to shorten the bunch and mitigate the hourglass effect.

In this article, we will first present the operational observations during the routine physics stores. Then we will analyze the mechanisms for the proton particle loss, transverse emittance growth, and bunch lengthening. Numerical calculations and models will then be presented to explain these observations.

The findings and conclusions of this article are important to understand the beam-beam effects in the past RHIC proton runs and could provide insight to improve the beam lifetime over the next RHIC luminosity upgrade program with head-on beam-beam compensation. However, the results from the latest successful $2015100 \mathrm{GeV}$ proton run including head-on beam-beam compensation with electron lenses are beyond the scope of this article.

\section{OPERATIONAL OBSERVATIONS}

\section{A. Beam loss}

The particle loss rate $\tau^{-1}(t)$ is used in the RHIC operation system, which is defined as

$$
\tau^{-1}(t)=-\frac{1}{N_{p}(t)} \frac{d N_{p}(t)}{d t},
$$

$N_{p}(t)$ is the instant beam intensity. The unit of particle loss rate is given in $\% / \mathrm{h}$. 

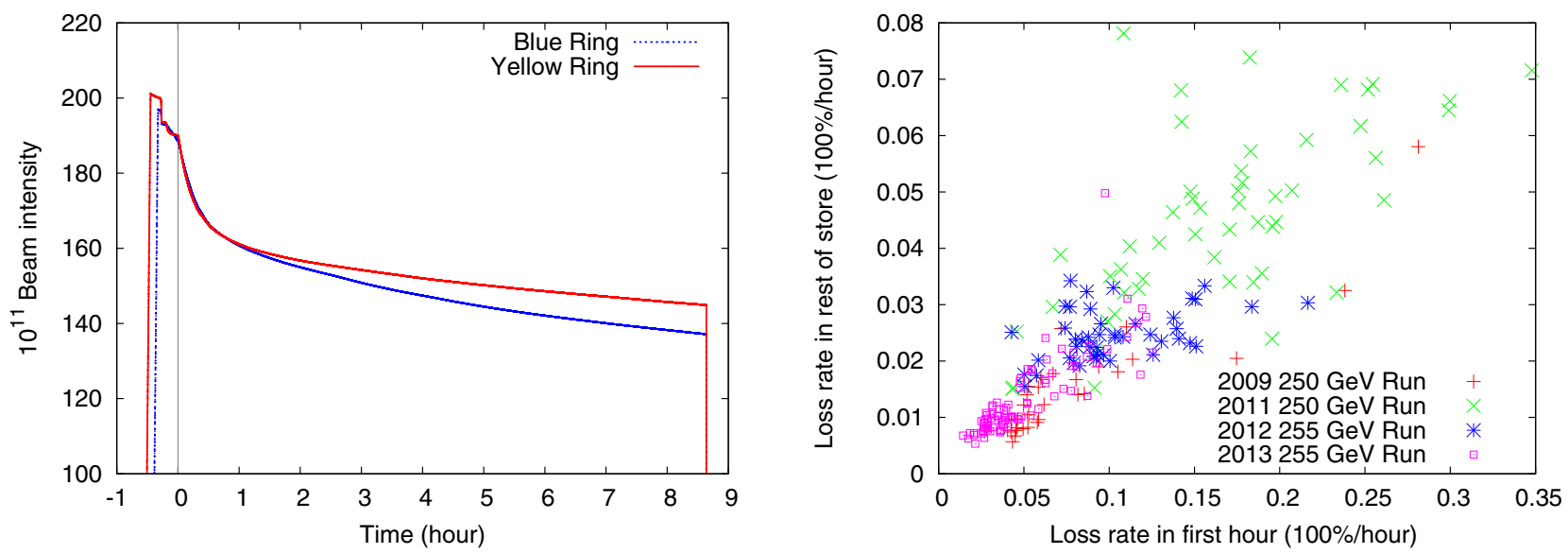

FIG. 3. Left: Evolutions of the beam intensities at store for Fill 16697. Right: Average particle loss rates in the first hour and in the rest of store for all the physics stores in the past $250 / 255 \mathrm{GeV}$ runs.

In the absence of collisions at the store energies, the particle loss rate could be less than $1 \% / \mathrm{h}$ depending on the machine tuning.

Right after we brought the two beams into collision by removing the vertical separation bumps at IP6 and IP8, the particle loss rate suddenly jumped up in the first 1-2 min. Then the particle loss rate slowly came down in the following 1-2 $\mathrm{h}$. After $2 \mathrm{~h}$ into store, the particle loss rate would stay below $5 \% / \mathrm{h}$.

As an example, the left plot of Fig. 3 shows the evolutions of the total beam intensities of both rings for Fill 16697 in the $2012255 \mathrm{GeV}$ run. The instant particle loss rate reached a maximum of $35 \% / \mathrm{h}$ in the Blue ring and $50 \% / \mathrm{h}$ in the Yellow ring right after collision. About 15\% of the total beam intensities were lost in the first hour of the store.

The right plot of Fig. 3 shows the average particle loss rates for all the physics stores in the past $250 / 255 \mathrm{GeV}$ runs. The horizontal axis is the average particle loss rate during the first hour and the vertical axis is the average particle loss rate during the rest of store. The particle loss rate in the first hour was 5 times larger than that for the rest of store. The beam loss rates in 2011 were much higher than other runs due to the commissioning of the $9 \mathrm{MHz}$ rf cavity and other systems.

The proton particle loss rate due to the luminosity burnoff is given by

$$
\tau_{\text {burn-off }}^{-1}=\frac{N_{\mathrm{IP}} L \sigma_{\mathrm{tot}}}{\left\langle N_{p}\right\rangle N_{B}}
$$

$\left\langle N_{p}\right\rangle$ is the average bunch intensity, $N_{B}$ the number of bunches per ring, and $N_{\text {IP }}$ the number of collisions per turn. $\sigma_{\text {tot }}$ is the total cross section of proton collision, which is 50 mbarn for the $250 \mathrm{GeV}$ proton collision. With the record luminosity $L=2.45 \times 10^{32} \mathrm{~cm}^{-2} \mathrm{~s}^{-1}$ in the $250 / 255 \mathrm{GeV}$ proton runs, together with $N_{p}=1.85 \times 10^{11}, N_{\mathrm{IP}}=2$, and $N_{B}=109$, we have

$$
\tau_{\text {burn-off }}^{-1}=0.44 \% / \mathrm{h} .
$$

Compared to the observed beam loss rates, the burn-off loss rate is negligible. This is a strong indication that the proton beam losses during the physics stores at RHIC are dominated by beam-beam effects.

\section{B. Transverse emittance and bunch length}

In RHIC, transverse emittances are measured with the ionization profile monitors (IPMs). Emittance measurement with the IPMs requires a good knowledge of the $\beta$ functions at the IPM location and a good calibration of the total 64 micro-channels which can lead to large uncertainties on the measurement.

The left plot of Fig. 4 shows the IPM measured normalized emittances throughout the store of Fill 16697. The horizontal and vertical emittances of both rings are shown. In the $2011250 \mathrm{GeV}$ and $2012255 \mathrm{GeV}$ proton runs, the transverse emittances normally first decreased in the first hour right after collision, then slowly increased in the rest of store. We associated the emittance reduction at the beginning of store to the fast large beam loss in the first hour. Fast loss of particles with large transverse amplitudes reduces the transverse rms beam sizes.

The wall current monitors (WCMs) are used to measure each bunch's longitudinal profile. The right plot of Fig. 4 shows the evolutions of rms bunch lengths of both rings for Fill 16697. The bunch lengths decreased in the first hour right after collision and then slowly increased over the rest of the store. Similarly to transverse emittance reduction, we associate the early bunch length reduction to the fast early beam losses, i.e., fast losses of particles with large momentum deviation reduces the rms bunch length. The spikes around $0.4 \mathrm{~h}$ in the plot are related to polarization measurements when the voltage of the $197 \mathrm{MHz}$ rf cavities was reduced to $70 \mathrm{kV}$.

During the $2013255 \mathrm{GeV}$ proton run, with the same lattices as in 2011 and 2012, a better beam lifetime was 

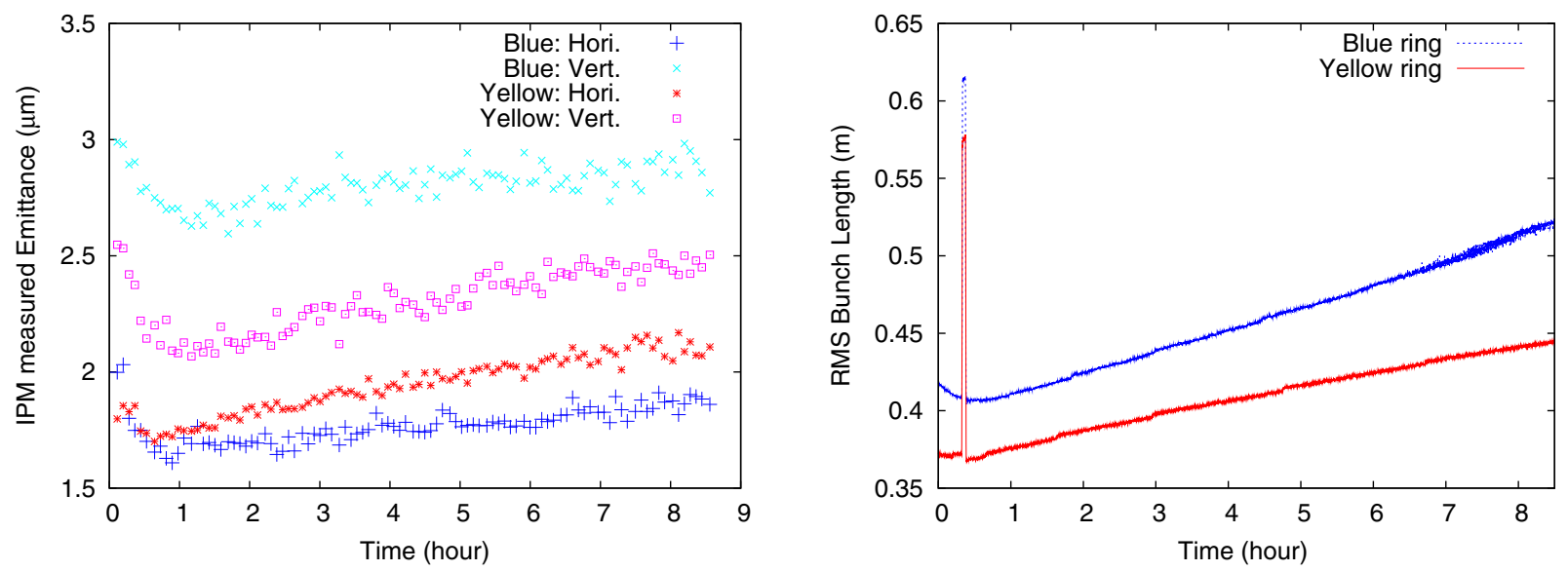

FIG. 4. Evolutions of the IPM measured transverse emittances (left) and the WCM measured rms bunch lengths (right) for both beams at store for Fill 16697.

obtained with a reduction of the voltage of the $197 \mathrm{MHz}$ cavities from previously $300 \mathrm{kV}$ to $100 \mathrm{kV}$. Another improvement in 2013 was the introduction of the longitudinal bunch-by-bunch dampers which were used at injection and on the energy ramp to mitigate the longitudinal instabilities. During the first hour of stores, the bunch length either remained stable or increased much slower than for the rest of store. We normally observed mild transverse emittance blowups right after collision which can be explained by a larger beam-beam parameter in that run. The transverse emittance then slowly increased over the rest of store.

For each run, after we entered the dedicated physics data taking operation mode, the initial beam parameters and their evolutions repeated very well from store to store. As an example, Fig. 5 shows the bunch lengths for all the physics stores in the 2012 and $2013255 \mathrm{GeV}$ runs. The horizontal axis is the initial bunch length at store. The vertical axis shows the bunch lengths at 1 and $6 \mathrm{~h}$ into store. The relative deviations of beam parameters between good

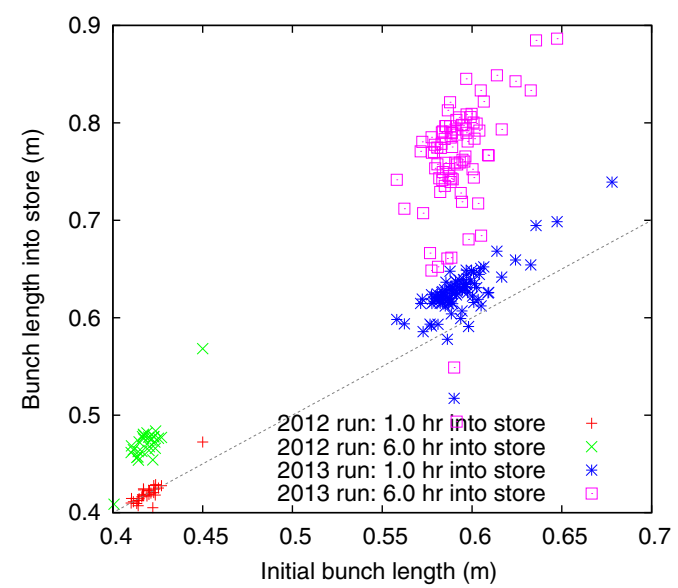

FIG. 5. Bunch lengths for all the physics stores in the 2012 and $2013255 \mathrm{GeV}$ runs. stores were less than $10 \%$. In the following analysis and simulation calculation, we will mainly focus on two typical stores: Fill 16697 from the $2012255 \mathrm{GeV}$ run with $300 \mathrm{kV}$ $197 \mathrm{MHz}$ cavities and Fill 17520 from the $2013255 \mathrm{GeV}$ run with $100 \mathrm{kV} 197 \mathrm{MHz}$ cavities.

\section{Store analysis}

To compare with the IPM emittance measurements and for further store analysis, according to Eq. (1), we define an average transverse emittance from the luminosity,

$$
\left\langle\epsilon_{L}\right\rangle=\frac{N_{p}^{2} N_{c} \gamma f_{\text {rev }}}{4 \pi \beta^{*} L} H\left(\beta^{*}, \sigma_{l}\right) .
$$

This luminosity-derived emittance cannot account for differences between the horizontal and vertical planes of the two rings but represents a more robust emittance measurement as it only depends on the luminosity and bunch intensity which are generally measured with high precision.

$H\left(\beta^{*}, \sigma_{1}\right)$ in Eq. (6) can be calculated precisely with the longitudinal bunch profiles. For an example, we slice the average longitudinal bunch profile in each ring into $K$ slices, then we have

$$
H\left(\beta^{*}, \sigma_{1}\right)=\sum_{j=1}^{K} \sum_{i=1}^{K} \frac{n_{1, i} n_{2, j} \sigma^{2}(0)}{N_{p, 1} N_{p, 2} \sigma^{2}(z)} .
$$

Here $n_{1, i}$ and $n_{2, j}$ are the particle populations of the $i$ th slice of beam 1 and of the $j$ th slice of beam 2. $N_{p, 1}$ and $N_{p, 2}$ are the bunch intensities of the two beams. $z$ is the longitudinal location where these two slices collide, $z=\left(z_{1, i}-z_{2, j}\right) / 2$, $z_{1, i}$ and $z_{2, j}$ are the relative longitudinal positions of the slices with respect to their bunch centers. $\sigma(0)$ and $\sigma(z)$ are the transverse beam sizes at IP and at the longitudinal location $z$ away from IP.

Figure 6 shows the calculated hourglass luminosity reduction factor and the luminosity-derived emittance for 


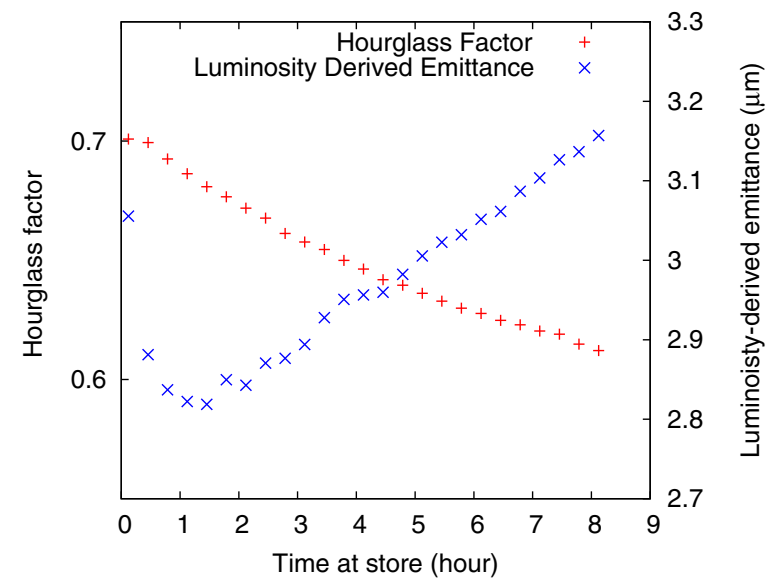

FIG. 6. Calculated hour-glass factor and luminosity-derived rms transverse emittance for Fill 16697.

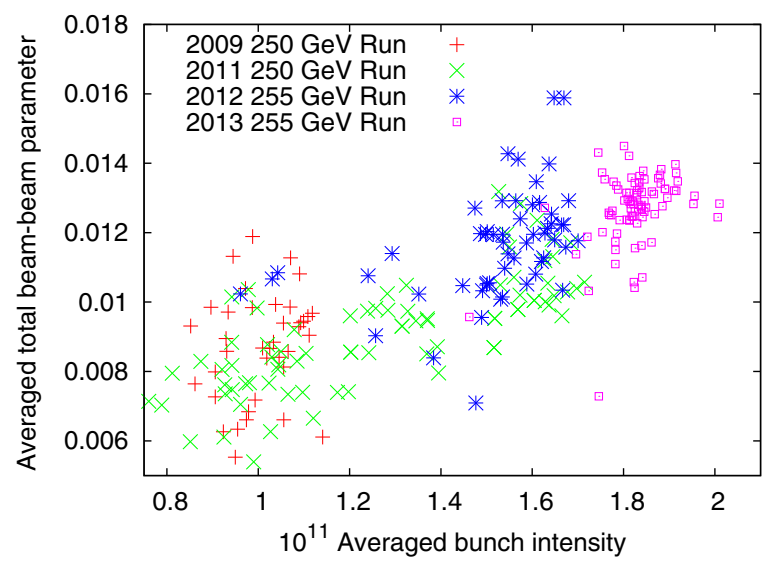

FIG. 7. Beam-beam parameters with 2 collisions versus the average proton bunch intensity in the past $250 / 255 \mathrm{GeV}$ runs.

Fill 16697. The hourglass factor decreased from 0.7 at the beginning to 0.6 at the end of the store. The luminosityderived emittance was decreasing in the first hour and then slowly increasing for the rest of store, which is in qualitative agreement with the IPM measurements.

With the luminosity-derived emittances, we can estimate the total beam-beam parameter with two collisions. Figure 7 shows the total beam-beam parameter at $5 \mathrm{~min}$ after collision for all the physics stores during the past $250 / 255 \mathrm{GeV}$ proton runs. Since we used average bunch intensities and emittances, the actual beam-beam parameter for each ring may slightly differ for each beam and plane depending on their respective beam parameters.

\section{MECHANISM OF BEAM LOSS}

\section{A. Correlations to beam loss}

We attempted to correlate the observed particle loss rates with the optics parameters such as the tunes, chromaticities, and the beam parameters such as the bunch intensity, transverse emittance, and beam-beam parameter. However, these correlations did not apply to the general cases, even though they might explain some specific observations of beam loss and/or emittance blowup. In this section we try to explain the general behavior of the machine in the case of ideal tuning. These specific cases of wrong tuning will therefore not be discussed.

As mentioned above, there are a few bunches only having 1 collision instead of 2 collisions per turn. The top-left plot of Fig. 8 shows the intensity evolutions of two adjacent bunches selected from Fill 16697: one bunch with 1 collision and the other one with 2 collisions per turn. These two bunches had very similar bunch intensities before collision. With collision, the particle loss rate for the bunch with 2 collisions was more than double that for the bunch with 1 collision.

The top-right plot of Fig. 8 shows the bunch lengths for the two adjacent bunches. The difference in their bunch lengths was very small at the beginning of store. There was not much bunch length growth in the first hour after collision. After that, the bunch lengths of both bunches began to grow. From the plot, the bunch length for the bunch with 1 collision per turn grew faster and got wider than the bunch with 2 collisions.

The bottom-left plot of Fig. 8 shows the longitudinal phase space with $360 \mathrm{kV} 28 \mathrm{MHz}$ and $300 \mathrm{kV} 197 \mathrm{MHz}$ rf voltages at the store energy $255 \mathrm{GeV}$. The horizontal axis is the distance to the center of the $28 \mathrm{MHz}$ rf bucket in units of ns. The central $\mathrm{rf}$ bucket of the $197 \mathrm{MHz}$ rf cavity is between [ $-2.5 \mathrm{~ns}, 2.5 \mathrm{~ns}]$. The vertical axis is the relative momentum deviation $d p / p_{0}$. The rf momentum acceptance for the combined $\mathrm{rf}$ systems is $1.1 \times 10^{-3}$. The typical longitudinal emittance of the proton beam in RHIC is $2.0 \mathrm{eV} \cdot \mathrm{s}$ and the relative rms beam momentum spread is about $2 \times 10^{-4}$.

From the bottom-left plot of Fig. 8, we can see that the particles away from the bunch center have a large momentum deviation. From the larger rms bunch length of the bunch with 1 collision we can deduce that the bunches with 1 collision per turn had a larger off-momentum dynamic aperture than the bunches with 2 collisions per turn.

The bottom-right plot of Fig. 8 shows the longitudinal bunch profiles over the store for the beam in the Blue ring of Fill16697. The profiles right after collision and 1 and $5 \mathrm{~h}$ with collision are shown. We notice that the particle intensity decreased dramatically in the central bunch area, especially during the first $1-2 \mathrm{~h}$, while the particle population and its distribution at the bunch tail did not.

Next we study the correlation between the particle loss and the particle movement in the longitudinal plane. For the moment, we focus on the change of particle population in the central bunch area $[-5 \mathrm{~ns}, 5 \mathrm{~ns}]$. For particles in this area, the relative momentum deviation is within $\left[-6 \times 10^{-4}, 6 \times 10^{-4}\right]$. Note that the area $[-5 \mathrm{~ns}$, 

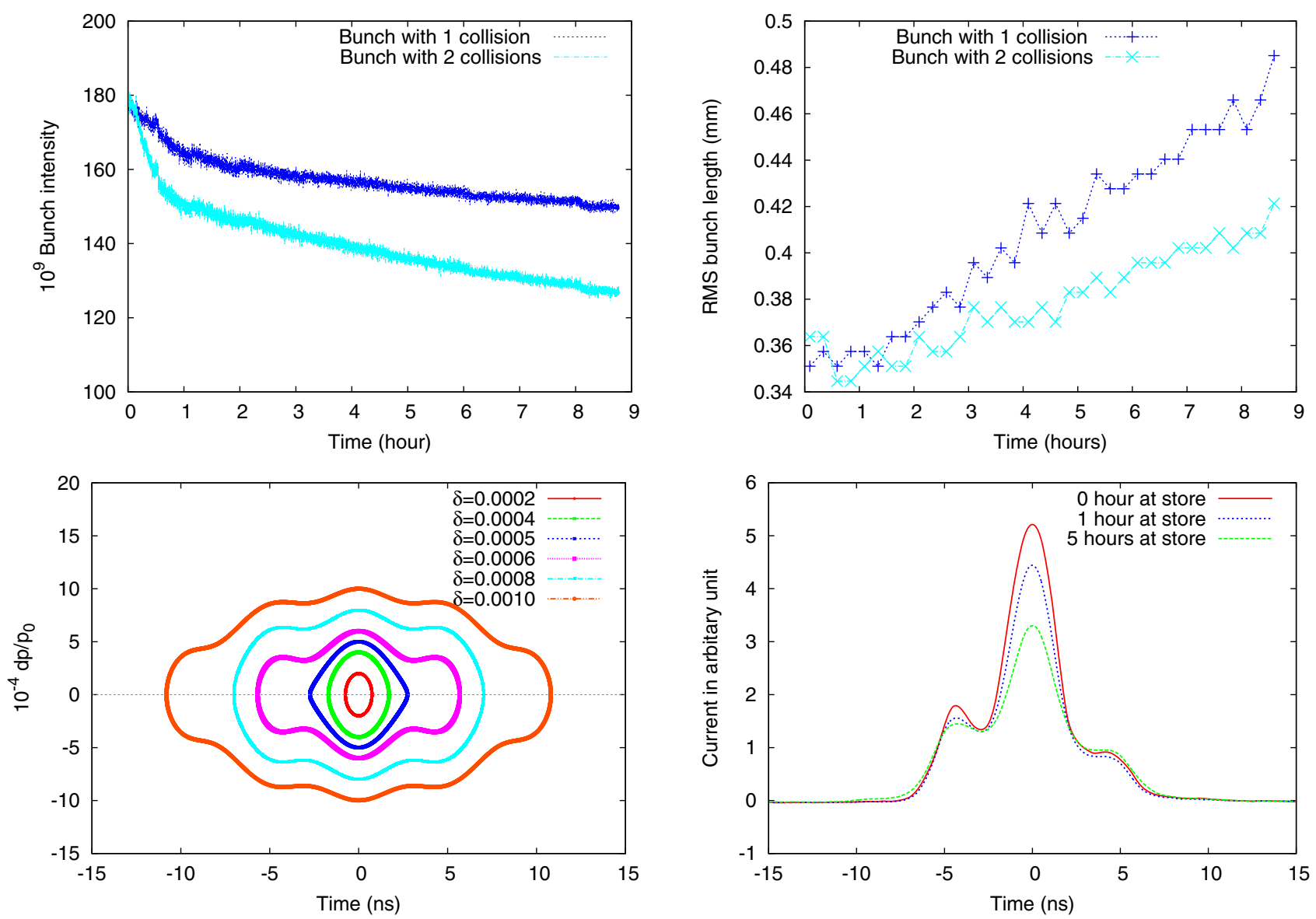

FIG. 8. Top-left: Intensity evolutions of two adjacent bunches, one with 1 collision per turn, the other 2 collisions per turn. Top-right: Bunch length evolutions of those two adjacent bunches. Bottom-left: Longitudinal phase space with $360 \mathrm{kV} 28 \mathrm{MHz}$ and $300 \mathrm{kV}$ $197 \mathrm{MHz}$ rf voltages. Bottom-right: The WCM measured longitudinal bunch profiles for the beam in the Blue ring of Fill 16697.

$5 \mathrm{~ns}$ ] is chosen arbitrarily. Choosing a different central region does not affect the following correlation.

The left plot of Fig. 9 shows the particle loss and particle leakage rates for all the bunches in the Blue ring for Fill
16697 over the whole bunch train during the first hour at store. The particle leakage rate is calculated from the population reduction in the area $[-5 \mathrm{~ns}, 5 \mathrm{~ns}]$ normalized by the initial total bunch intensity. From this plot, we found
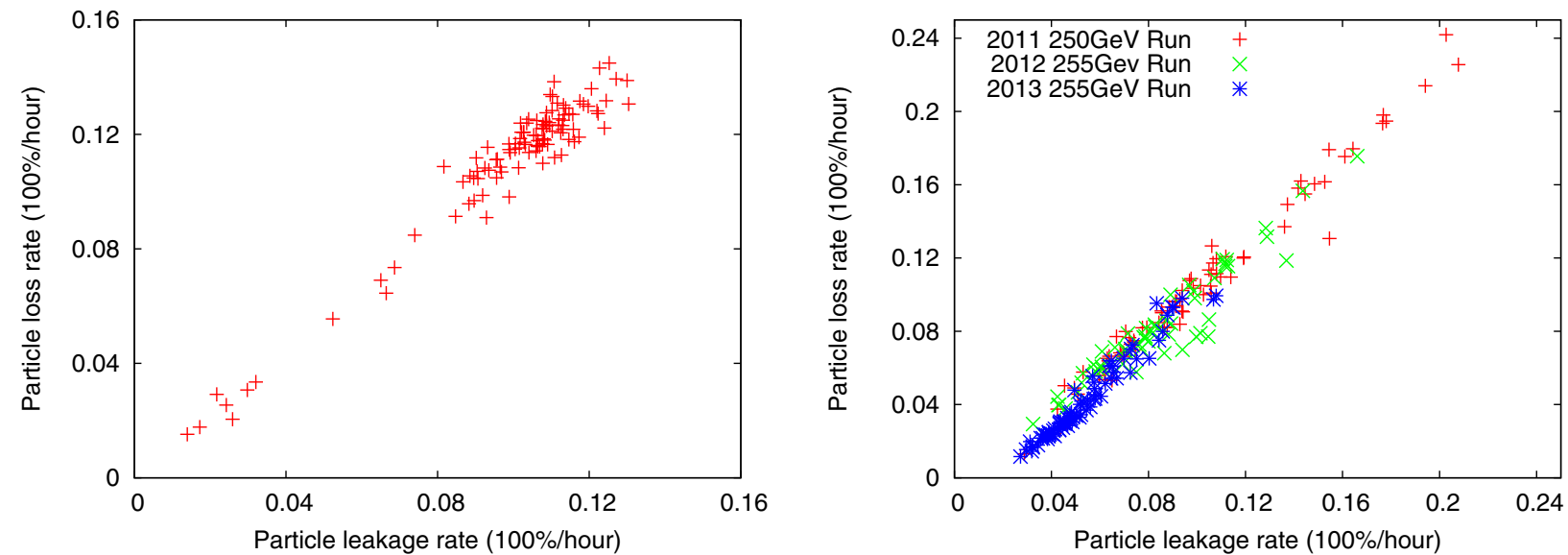

FIG. 9. Left: Correlation between the particle loss rate and the particle leakage rate during the first hour of store for all the bunches of Fill 16697. Right: Correlation between the particle loss rate and the particle leakage rate during the first hour of store for the beam in the Blue ring for all the physics stores in the past $250 / 255 \mathrm{GeV}$ proton runs. 
a strong linear correlation between the particle loss rates and particle leakage rates for all the bunches during the first hour of collision. The few points in the bottom-left corner of the plot represent the bunches with 1 collision per turn which have slower losses.

The above linear correlation between the particle loss and the particle leakage for all bunches also holds for other stores, and for any period of any given store. Furthermore, if we use the average WCM bunch profile and the total beam intensity loss, a similar linear correlation exists. The right plot of Fig. 9 shows the linear correlation between the total particle loss and the total particle leakage rates during the first hour at store for the Blue ring for all the physics stores in the past $250 / 255 \mathrm{GeV}$ proton runs.

During the routine physics operation, we did not observe debunched beam from the WCMs. For this reason, all the particles lost originate from the bunched beam and were lost in the transverse planes. Considering that the particle loss rate is proportional to the number of particles moving out of the central bunch area and the particles in the bunch tails have large momentum deviations, we correlate the particle loss to a limited transverse off-momentum aperture. In addition, the large beam losses only happened with collision. We conclude that the proton beam loss in the routine physics stores is due to a limited off-momentum dynamic aperture which is reduced by the beam-beam interactions.

\section{B. Off-momentum dynamic aperture}

In this section, we compute and compare the offmomentum dynamic apertures with and without beambeam interactions. We track particles in a 6-dimensional phase space $\left(x, p_{x}, y, p_{y}, c \Delta t, \delta=d p / p_{0}\right)$ up to $10^{6}$ turns with SimTrack $[15,16]$. The initial particles are launched in 10 equally spaced directions in the first quadrant of the $\left(\sqrt{2 J_{x} \beta_{x}} / \sigma_{x}, \sqrt{2 J_{x} \beta_{y}} / \sigma_{y}\right)$ plane, $\beta_{x, y}$ are the $\beta$-functions at the starting point of the lattice. The dynamic aperture is given in units of the transverse rms beam size $\sigma$. We only compare the minimum dynamic apertures between different beam-beam conditions.

As an example, we look at the lattices used during the $255 \mathrm{GeV}$ proton runs. The $\beta^{*} \mathrm{~s}$ at the collision points are $0.65 \mathrm{~m}$. The lattice tunes are $(28.690,29.685)$, which are close to the operational settings. The linear chromaticities are corrected to $(1,1)$ before tracking. The second order chromaticities are $(2100,4400)$ for the Blue ring and $(-6100,-1700)$ for the yellow ring. Second order chromaticities are defined as $\xi_{x, y}^{(2)}=\frac{1}{2} \frac{\partial^{2} Q_{x, y}}{\partial \delta^{2}}, \delta=d p / p_{0} .360 \mathrm{kV}$ for the $28 \mathrm{MHz}$ rf cavities and $300 \mathrm{kV}$ for the $197 \mathrm{MHz}$ rf cavities are used. The normalized rms transverse emittance is $3.3 \mu \mathrm{m}$. The bunch intensity is $1.8 \times 10^{11}$, which gives a total beam-beam parameter of 0.0135 .

Figure 10 shows the calculated off-momentum dynamic apertures versus the relative momentum deviation $d p / p_{0}$

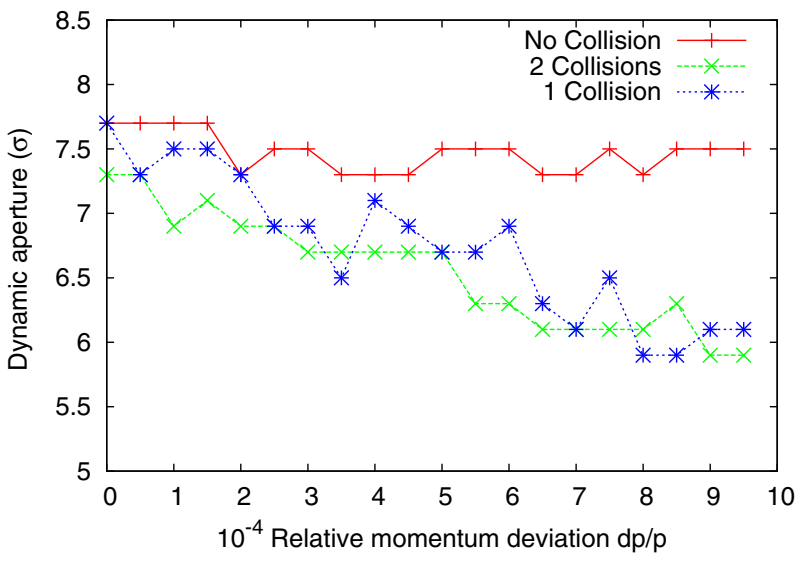

FIG. 10. Calculated off-momentum dynamic apertures without and with beam-beam interactions for the Blue ring. The particle energy is $255 \mathrm{GeV}$.

for the Blue ring. Three cases are studied: without collision, with 2 collisions at IP6 and IP8, and with 1 collision at IP6. The dynamic aperture without collision stays around $7.5 \sigma$ for $d p / p_{0}$ between 0 and $1.0 \times 10^{-3}$. Beam-beam interactions reduce the dynamic aperture: the larger the momentum deviation, the smaller the dynamic aperture. For $d p / p_{0}$ equals $7 \times 10^{-4}$, the dynamic aperture with collision is approximately $6 \sigma$, which is $1.5 \sigma$ less than the case without collision. For most of the cases shown in Fig. 10, the dynamic aperture with 1 collision is slightly larger than the one with 2 collisions.

Figure 11 shows the off-momentum dynamic apertures for the Blue ring with and without beam-beam interactions as a function of tunes. The tunes are kept below the diagonal and between $2 / 3$ and $7 / 10$. The horizontal axis is the vertical fractional tune. The horizontal fractional tune is kept at 0.005 above the vertical one. The same beam parameters as in the above dynamic aperture calculation are used. The initial relative momentum deviation is set to

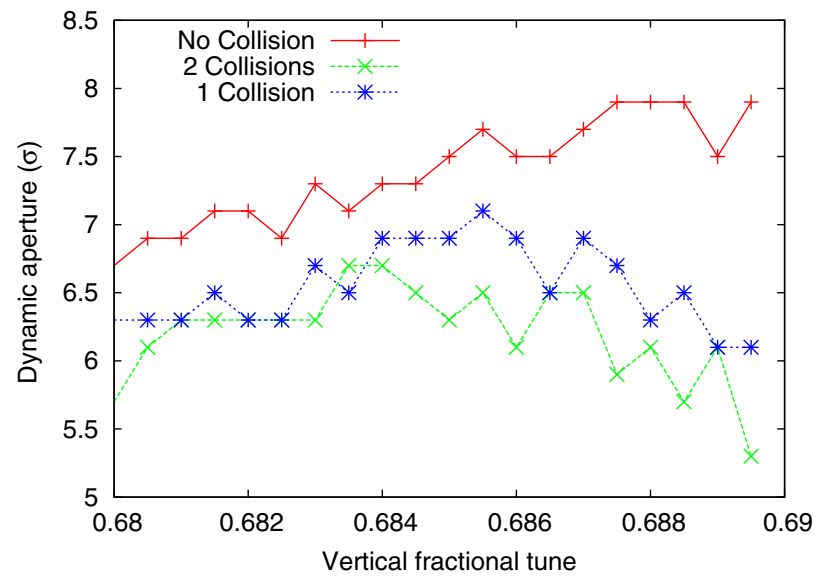

FIG. 11. Calculated off-momentum dynamic apertures for the Blue ring in a tune scan along the diagonal in the tune space between $2 / 3$ and $7 / 10$. The particle energy is $255 \mathrm{GeV}$. 
$6 \times 10^{-4}$, which is 3 times the rms relative momentum deviation.

From Fig. 11, the case without collision gives the largest dynamic aperture among the three cases studied. The dynamic aperture without collision decreases when the working point moves toward the third order resonance. For the cases with collision, the dynamic aperture drops when the working point moves toward $2 / 3$ or $7 / 10$. For the operational working point with the vertical tune between 0.684 and 0.690 , the case with 1 collision per turn gives a larger dynamic aperture than the case with 2 collisions per turn.

To summarize, numerical calculation of dynamic apertures verifies that the beam-beam interactions in the RHIC proton operation reduces the off-momentum dynamic aperture. And the bunches with 1 collision per turn have a slightly larger dynamic aperture than the bunches with 2 collisions.

\section{MECHANISM OF EMITTANCE GROWTH}

\section{A. Beam-gas scattering}

In RHIC, the most important beam-gas interactions are the inelastic nuclear collisions and the elastic nuclear Coulomb scatterings between the protons and the nuclei of gas atoms [17]. The inelastic nuclear collisions cause proton losses and reduce the beam lifetime, while the multiple elastic nuclear Coulomb scatterings cause proton beam emittance growth.

The cross section of the nuclear collision can be estimated as [18]

$$
\sigma_{N} \simeq \pi R_{N}^{2},
$$

with $R_{N}=r_{0}\left(A_{i}^{1 / 3}+A_{t}^{1 / 3}\right), r_{0} \simeq 1.2 \mathrm{fm}, A_{i, t}$ are the atom mass numbers of the projectile ions and the target gas atoms. The cross sections between the proton and the residual gas atoms are in an order of $10^{-25} \mathrm{~cm}^{-2}$.

In standard operation, $83 \%$ of the beam pipe is at $4.5 \mathrm{~K}$ with a pressure of $0.01 \mathrm{nTorr}$. The remaining is at room temperature, $300 \mathrm{~K}$, with a pressure of $0.5 \mathrm{nTorr}$. The warm sections are mainly located in the 6 IRs. The residual gas is made of $100 \% \mathrm{H}_{\mathrm{e}}$ gas in the cold regions, and $95 \% \mathrm{H}_{2}$ and 5\% $\mathrm{CO}$ in the warm regions. Based on these vacuum parameters, the beam-gas lifetime of the RHIC protons is $1250 \mathrm{~h}$ and thus negligible compared to other sources.

The cross section of single elastic Coulomb scatterings between protons and the nuclei of gas atoms is given by the Rutherford cross section [18]. The averaged square of the scattering angle $\theta$ is

$$
\left\langle\theta^{2}\right\rangle=2 \theta_{\min }^{2} \ln \left(\frac{\theta_{\max }}{\theta_{\min }}\right)=4 \theta_{\min }^{2} \ln \left(204 Z_{t}^{-1 / 3}\right),
$$

where $\theta_{\min }$ is the minimum scattering angle due to screening of electrons of target atoms, $\theta_{\max }$ the maximum scattering angle due to finite sizes of target nuclei, $Z_{t}$ the atom number of the target atom. For the RHIC proton operation, $\sqrt{\left\langle\theta^{2}\right\rangle}$ is of the order of $10^{-8}-10^{-7} \mathrm{rad}$. The rms angle spread $\sigma_{x^{\prime}}$ of the proton beam is of the order of $10^{-5} \mathrm{rad}$. Therefore, single elastic Coulomb scattering cannot cause proton loss.

The growth rate of the rms normalized emittance of the proton beam due to multiple elastic Coulomb scatterings between protons and the nuclei of gas atoms is calculated with [19]

$$
\frac{d \epsilon_{n, \mathrm{rms}}}{d t}=2 \pi \gamma_{i}\langle\beta\rangle n_{t} \beta_{i} c\left(\frac{2 Z_{i} Z_{t} r_{p}}{A_{i} \beta_{i}^{2} \gamma_{i}}\right)^{2} \ln \left(204 Z_{t}^{-1 / 3}\right),
$$

where $\beta_{i} c$ and $\gamma_{i}$ are the velocity and Lorentz factor of the protons, $n_{t}$ the density of gas atoms. $\langle\beta\rangle$ is the average $\beta$ function along the ring, which is $45 \mathrm{~m}$ in the cold sections and $115 \mathrm{~m}$ in the warm sections.

For the $100 \mathrm{GeV}$ RHIC protons, the calculated normalized rms emittance growth due to multiple elastic Coulomb scatterings is $4.9 \times 10^{-3} \mu \mathrm{m} / \mathrm{h}$. And for the $250 / 255 \mathrm{GeV}$ RHIC protons, it is $2.5 \times 10^{-3} \mu \mathrm{m} / \mathrm{h}$. Therefore, the emittance growth due to the beam-gas interaction can be neglected too.

\section{B. Touschek effect}

Coulomb scattering of charged particles in a bunched beam results is an exchange of energy between different degrees of freedom [20]. Usually two scattering regimes are considered: the single scattering when a rare single largeangle scattering makes a large change to the particle's momentum (Touschek effect $[21,22]$ ), and the multiple small-angle scatterings which lead to a diffusion process (Intrabeam scattering or IBS effect [23-25]). Touschek effect can cause particle loss and create non-Gaussian bunch tails. IBS is usually responsible for the changes of particle distribution in the bunch core and hence emittance growth.

The particle loss rate $\tau_{T}^{-1}\left(\delta_{m}\right)$ due to the Touschek effect for a 3-d Gaussian distribution is given in Ref. [20]. $\delta_{m}$ is the maximum momentum aperture, which is assumed to be much larger than the beam's momentum spread. Here we use these equations to estimate the particle leakage rate out of the central bunch area due to the Touschek effect. For an example, we again look at the central area $[-5 \mathrm{~ns}, 5 \mathrm{~ns}]$.

Considering the fact that particles with different initial momentum deviation need different momentum change to be able to leak out of the central bunch area, the total leakage rate is [26]

$$
\tau_{\text {leakage }}^{-1}=\int_{0}^{6 \times 10^{-4}} \tau_{T}^{-1}\left(6 \times 10^{-4}-\delta\right) \rho(\delta) d \delta .
$$

$\rho(\delta)$ is the distribution of relative momentum deviation for particles in the area $[-5 \mathrm{~ns}, 5 \mathrm{~ns}]$. 


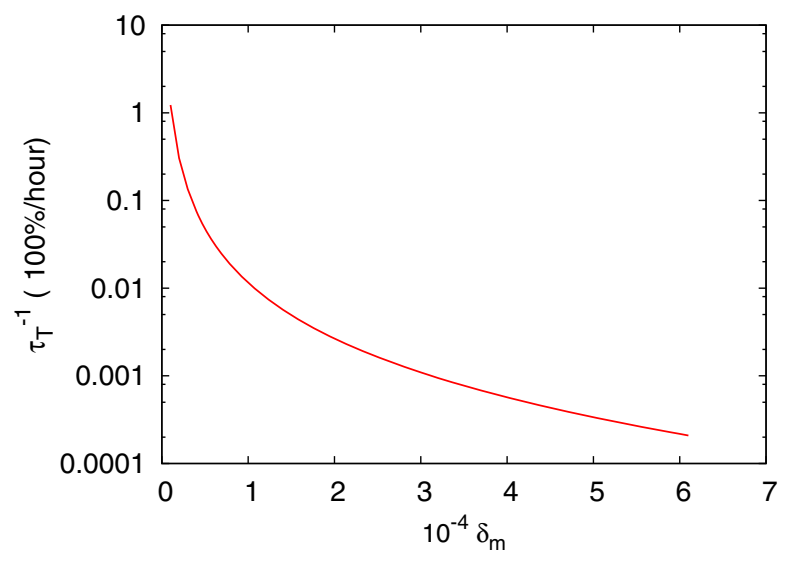

FIG. 12. The percentage of protons per hour having a relative momentum change $\delta_{m}$ due to Touschek effect.

For simplicity, we assume a Gaussian distribution of particles in the bunch central area. For Fill 16697, the bunch intensity is $1.8 \times 10^{11}$. However, only about $1.35 \times 10^{11}$ particles are in the central Gaussian distribution. The rms bunch length was $0.4 \mathrm{~m}$, and the relative rms momentum deviation was $2 \times 10^{-4}$. Figure 12 shows $\tau_{T}^{-1}$ as a function of $\delta_{m}, \delta_{m}$ is scanned from $1.0 \times 10^{-5}$ to $6 \times 10^{-6}$ with a step size of $1.0 \times 10^{-5}$.

According to Eq. (13), the calculated particle leakage rate out of the central area $[-5 \mathrm{~ns}, 5 \mathrm{~ns}]$ is about $1.1 \% / \mathrm{h}$, which is about 10 times smaller than the actual observations. As we reported earlier, more than $10 \%$ of the total number of particles leaked out of the central area $[-5 \mathrm{~ns}$, $5 \mathrm{~ns}$ ] during the first hour of the stores during the 2012 $255 \mathrm{GeV}$ run. Therefore, the Touschek effect is not the main source to particles moving out of the central bunch area.

\section{IBS effect}

The exact equations to calculate the IBS growth rates for a 3-d Gaussian distribution bunch are given in Ref. [20]. For a quick estimate, we use an approximate approach given in Ref. [27]. The difference in the growth rates between the exact and approximate calculations is within $20 \%$. The IBS growths rates from the approximate approach are given by

$$
\begin{gathered}
\tau_{\mathrm{IBS}, \|}^{-1}=\frac{1}{\sigma_{p}^{2}} \frac{d \sigma_{p}^{2}}{d t}=\frac{r_{i}^{2} c N_{i} \Lambda}{8 \beta^{3 / 2} \gamma^{3 / 2} \epsilon_{n, \mathrm{rms}, x}^{3 / 2}\left\langle\beta_{x}^{1 / 2}\right\rangle \sigma_{l} \sigma_{p}^{2}}, \\
\tau_{\mathrm{IBS}, x, y}^{-1}=\frac{1}{\epsilon_{n, \mathrm{rms}, x, y}} \frac{d \epsilon_{n, \mathrm{rms}, x, y}}{d t}=\frac{1}{2} \frac{\gamma \beta \sigma_{p}^{2}}{\epsilon_{n, \mathrm{rms}, x}}\left\langle\frac{H_{x}}{\beta_{x}}\right\rangle \tau_{\|}^{-1} .
\end{gathered}
$$

Here $\sigma_{p}$ is the rms relative momentum spread, $\sigma_{l}$ is the rms bunch length, and $\epsilon_{n, \mathrm{rms}, x}$ is the transverse normalized rms emittance. $\Lambda$ is the Coulomb logarithm. $H_{x}=\left(D_{x}^{\prime} \beta_{x}+\right.$ $\left.\alpha_{x} D_{x}\right)$ is the invariant of dispersion, $D_{x}$ is the horizontal dispersion, $\alpha_{x}$ is the horizontal alpha-function of Twiss parameters. Here we assumed the same emittance growth rates for the horizontal and vertical planes, which is the case for the standard RHIC operation.

For RHIC proton lattices with $\beta^{*}=0.65 \mathrm{~m}$ at IP6 and IP8, we have $\left\langle\beta_{x}\right\rangle=6.7 \mathrm{~m}$ and $\left\langle H_{x} / \beta_{x}\right\rangle=0.045$. For Fill 16697, at the beginning of the store, the bunch intensity was $1.7 \times 10^{11}$, the rms normalized emittance $2.5 \mu \mathrm{m}$, the rms bunch length $0.4 \mathrm{~m}$, and the rms relative momentum spread $1.4 \times 10^{-4}$, the calculated IBS growth times are $1.3 \mathrm{~h}$ in the longitudinal plane and $26.6 \mathrm{~h}$ in the transverse plane. The longitudinal IBS growth rate is much faster than the transverse emittance growth.

If we assume that there is no dynamic aperture limit and no particle loss, the momentum spread and the bunch length would increase by $39 \%$ while the transverse emittance only increases by $4 \%$ in the first hour of stores. Based on the rms bunch length change, we calculate that there would be $11 \%$ of the total number of particles moving out of the area [-2.5 ns, $2.5 \mathrm{~ns}]$ during the first hour. Based on the WCM measurements, there was $10 \%$ of total particles moving out of the area [-2.5 ns, $2.5 \mathrm{~ns}]$.

During the first hour at store with collision, there were $15 \%$ of the total beam loss for Fill 16697. IBS not only moved the particles from the central $197 \mathrm{MHz}$ rf bucket to the bunch tail but also increased the off-momentum deviations of the particles initially in the bunch tail. Due to the reduced off-momentum dynamic aperture with beam-beam interactions, particles in the bunch tail with large off-momentum deviations would get lost.

After the first hour at store, the IBS growth rates were smaller due to the reduced bunch intensity and increased bunch length and momentum spread. For Fill 16697, the calculated IBS growths rates are $3.4 \mathrm{~h}$ in the longitudinal plane and $39.0 \mathrm{~h}$ in the transverse plane. We calculate that there would be $4 \%$ of the total number of particles moving out of the area [ $-2.5 \mathrm{~ns}, 2.5 \mathrm{~ns}]$ during the second hour. Based on the WCM measurements, there were $3.8 \%$ of the total number of particles moving out of the area $[-2.5 \mathrm{~ns}$, $2.5 \mathrm{~ns}]$. The actual total beam loss during the second hour at store is $5 \% / \mathrm{h}$ for Fill 16697 . The particle population in the bunch tail almost remained stable.

To summarize, IBS is the main cause to move the particles out of the bunch central area. The particles in the bunch tail had large off-momentum deviations and would get lost with beam-beam interactions. As discussed earlier, beam-beam interactions reduce the off-momentum dynamic aperture.

\section{NUMERICAL MODELING AND MULTIPARTICLE TRACKING}

\section{A. Numerical modeling of emittance growth}

In this section, we numerically model the transverse emittance growth and bunch lengthening at store due to 
the IBS effect. For simplicity, we assume a 3-d Gaussian distribution for the particles in the bunch central area and use Eqs. (14) and (15) to calculate the growth rates of the transverse emittance and bunch length. The results are to be compared with the actual measurements.

In the code, we update the IBS growth rates and the transverse emittance and bunch length every $20 \mathrm{~s}$. The initial inputs are the average bunch intensity, the WCM measured bunch length, and the luminosity-derived transverse emittance at the beginning of store. The real evolution of the bunch intensity throughout the store is used.

First we model the bunch length growth without beambeam interaction. As an example, Fig. 13 shows the measured and IBS-predicted rms bunch lengths for the beam in the Yellow ring for Fill 16715. This short store was used for the proton polarization lifetime measurement and there was no collision. The voltage of the $197 \mathrm{MHz}$ rf cavity was $60 \mathrm{kV}$ and the longitudinal bunch profile was Gaussian. The initial bunch intensities was $0.8 \times 10^{11}$, the rms bunch length was $0.56 \mathrm{~m}$, and the rms relative momentum spread was $2 \times 10^{-4}$. A typical normalized rms transverse emittance $3 \mu \mathrm{m}$ at the beginning of store was assumed since we did not have a good IPM emittance measurement for this store. During the 4-h long store, the beam loss rate was less than $1 \% / \mathrm{h}$ except during the polarization measurements.

From Fig. 13, the IBS effect largely predicted the trend of the actual bunch lengthen increase, especially during the first hour. There were two polarization measurements at 1 and $3 \mathrm{~h}$ into store which introduced additional bunch length increase. The difference in the bunch lengths between the IBS prediction and the measurement is less than $5 \%$ at the end of store. The difference in the net bunch length increases in $4 \mathrm{~h}$ is about 20\%. From Fig. 13, these two polarization measurements accounted for the most difference between the prediction and the measurement.

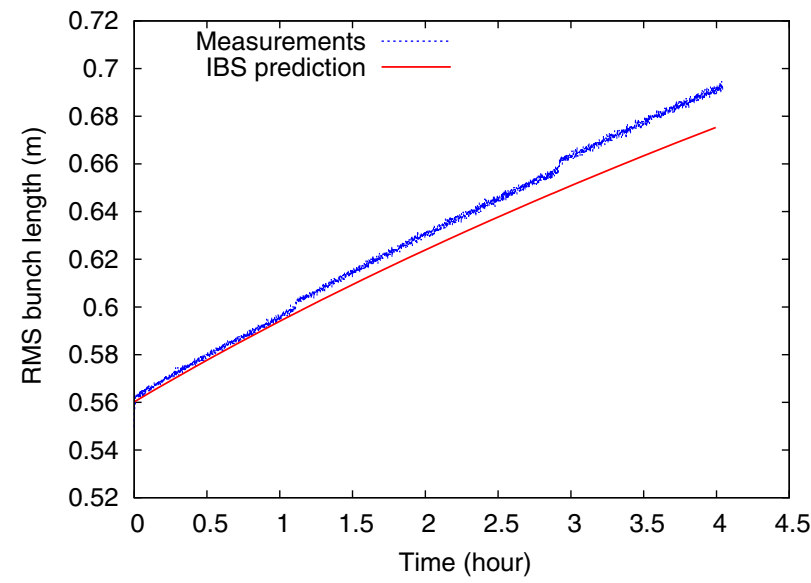

FIG. 13. Numerical modeling of bunch length growth without collision at store. Initial beam parameters similar to Fill 16697 are used.
Next we numerically model the emittance growth and bunch lengthening for Fill 16697 which includes collisions. As discussed above, the reductions in the emittances and bunch lengths at the beginning of store are related to a fast large intensity loss. Our modeling therefore starts at $1.5 \mathrm{~h}$ into the store. Again we assume a 3-d Gaussian distribution for the particles in the central bunch area. Based on the WCM longitudinal profile measurement, about $70 \%$ of the total particles are in the central Gaussian distribution.

For this fill, the initial bunch intensity in the central Gaussian distribution was $0.85 \times 10^{11}$, the $\mathrm{rms}$ bunch length was $0.42 \mathrm{~m}$, and the initial relative rms momentum spread was $3 \times 10^{-4}$. The initial normalized rms transverse emittance was $2.8 \mu \mathrm{m}$, which was derived from luminosity. The real bunch intensity evolution is used here too.

Figures 14 and 15 show the IBS-predicted transverse emittance growth and rms bunch lengthening for the beam in the Blue ring and their measured values. The difference in the emittance growth between the IBS prediction and

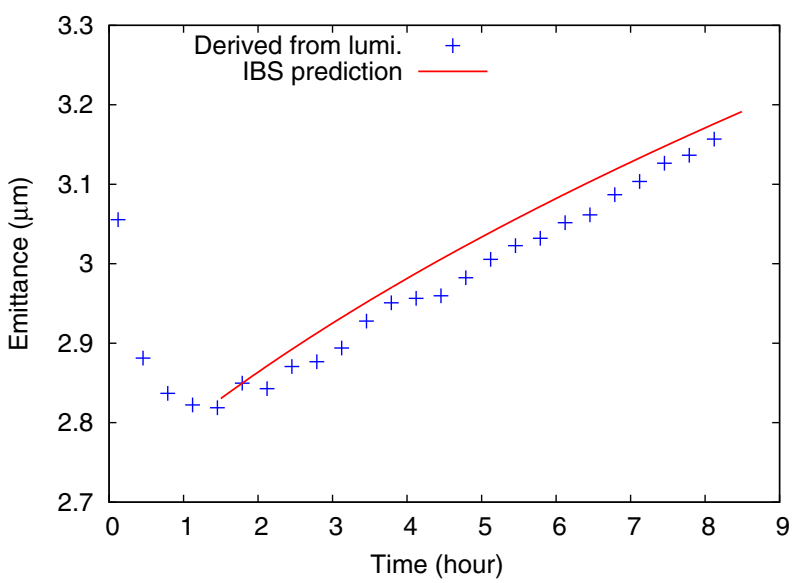

FIG. 14. Numeric modeling of emittance growth with collision at store. Initial beam parameters similar to Fill 16697 are used.

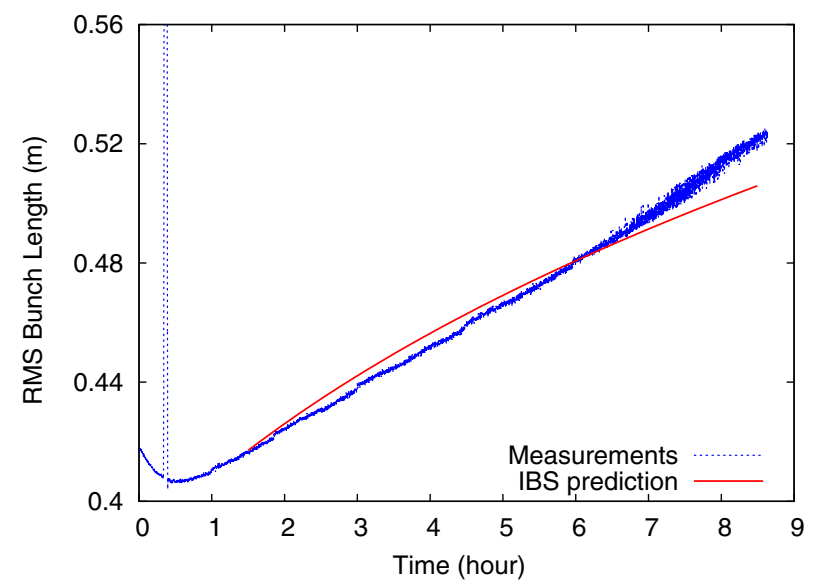

FIG. 15. Numerical modeling of bunch length growth with collision at store. Initial beam parameters similar to Fill 16697 are used. 
the measurement is below 5\%. The difference in the net bunch length increase in $7 \mathrm{~h}$ at store is about $10 \%$. These differences may come from our 3-d Gaussian bunch assumption in the simulation. And we also ignored all other effects except the IBS effect. As mentioned above, Touschek effect moves particles out of the bunch center and generates a non-Gaussian distribution tail. The method to measure the average bunch length with WCMs also gives some systematic errors since the differences between bunches are ignored.

\section{B. Multiparticle tracking 1. Simulation code}

Multiparticle numerical simulation was carried out to understand and model the evolutions of longitudinal bunch profiles and particle loss for non-Gaussian proton bunches at store. In the simulation, the transverse motion is represented by a $4 \times 4$ linear matrix and the transverse chromaticities are set to 2 . Both the $28 \mathrm{MHz}$ and $197 \mathrm{MHz}$ rf cavities are included. The time-of-flight is calculated with the slippage factor.

To generate the non-Gaussian longitudinal bunch profile at store, we first generate a 6-d Gaussian distribution of macroparticles with $360 \mathrm{kV} 28 \mathrm{MHz}$ rf voltage. Then we slowly ramp up $300 \mathrm{kV} 197 \mathrm{MHz}$ rf voltage. After a few seconds a stable longitudinal distribution is obtained, we begin to add the IBS and beam-beam effects. In this study, half a million of macroparticles are used.

To simulate the IBS growth for non-Gaussian bunch, we slice the proton bunch into 100 slices longitudinally. For each slice, we estimate its IBS growth rates with the formula for the coasting beam. To save the computing time, enlarged Gaussian random IBS kicks are applied each turn to the macroparticles. This method had been benchmarked with the analytical predictions for a Gaussian distribution bunch and was successfully used for the stochastic cooling simulation for the RHIC ion operation [28].

In our study, we simply apply a hard momentum aperture cut to represent the effect of beam-beam interactions. If a macroparticle's off-momentum is larger than it, this particle will be immediately eliminated and considered lost. The burn-off caused particle loss is also included by changing the weights of macroparticles based on their longitudinal positions in the bunch.

\section{Simulation results}

Multiparticle simulation largely reproduces the evolutions of longitudinal bunch profiles and transverse emittances at store. As an example, Fig. 16 shows the longitudinal bunch profiles with particle loss caused by burn-off only. The initial beam parameters were similar to Fill 16697. The particles continue to move out of the central $197 \mathrm{MHz}$ rf bucket due to the IBS effect. Since there is no

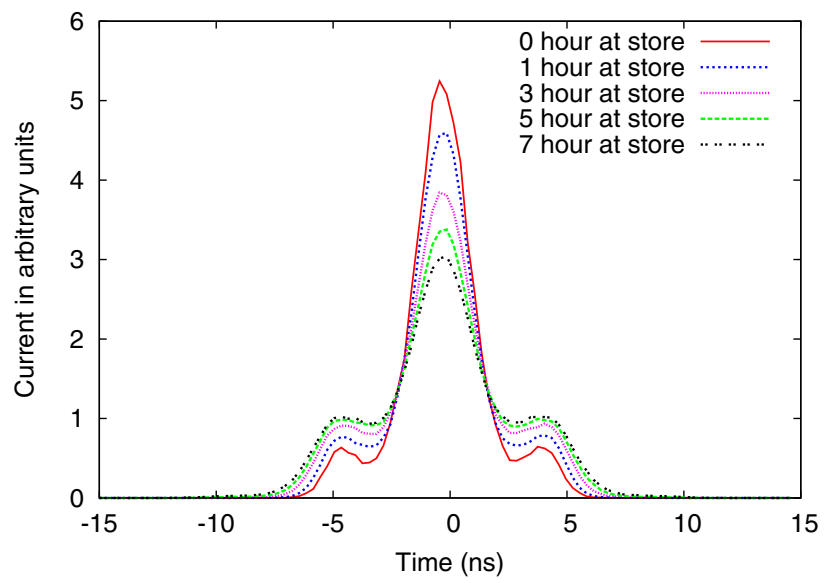

FIG. 16. Longitudinal bunch profiles at store from multiparticle simulation with $300 \mathrm{kV} 197 \mathrm{MHz}$ rf cavities. Momentum aperture is not included.

momentum aperture limit, the tail population continues to increase over the store.

Figure 17 shows the longitudinal bunch profiles with an off-momentum aperture $6.0 \times 10^{-4}$. Particles in the bunch tail with an off-momentum deviation larger than the momentum aperture will get lost. In less than an hour a stable particle distribution has been created in the bunch tail. In the study, if the momentum aperture is set to be $7.0 \times 10^{-4}$ or larger, the tail population will continuously increase over the store. If the momentum aperture is set to be smaller than $6.0 \times 10^{-4}$, a stable particle distribution still can be generated in the bunch tail but with a smaller population than the beginning of store. Compared to the measured bunch profiles as shown in the bottom-right plot of Fig. 8, the actual momentum aperture in the RHIC proton operation is likely around $6.0 \times 10^{-4}$.

Furthermore, Fig. 18 shows the longitudinal bunch profiles at store from the multiparticle tracking with

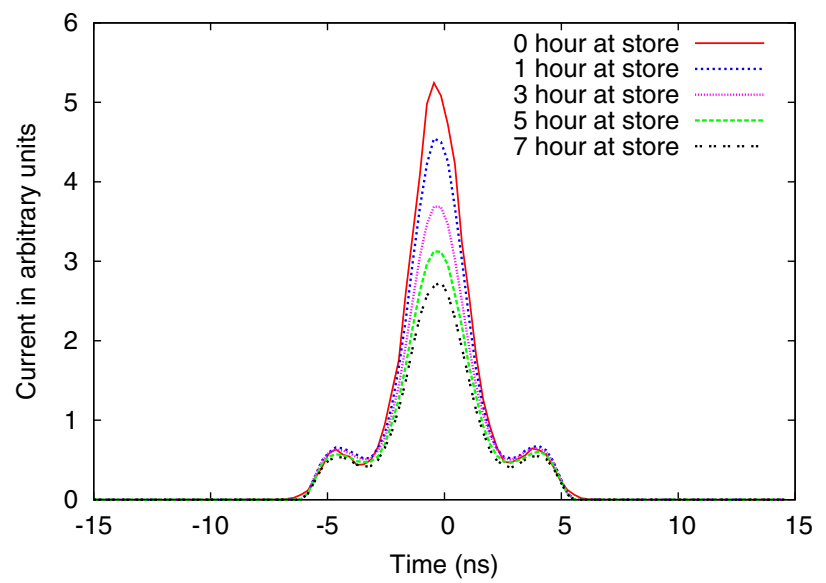

FIG. 17. Longitudinal bunch profiles at store from multiparticle simulation with $300 \mathrm{kV} 197 \mathrm{MHz}$ rf cavities. Momentum aperture $6 \times 10^{-4}$ is applied. 


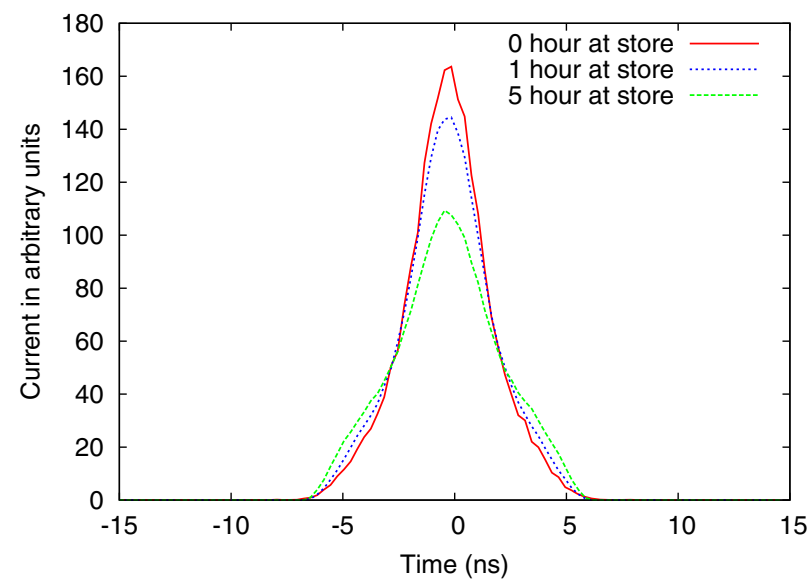

FIG. 18. Longitudinal bunch profiles at store from multiparticle simulation with $100 \mathrm{kV} 197 \mathrm{MHz}$ rf cavities. Momentum aperture $6 \times 10^{-4}$ is applied.

$100 \mathrm{kV} 197 \mathrm{MHz}$ rf cavities which was used in 2013. The initial beam parameters are from Fill 17520. The profiles from simulation qualitatively agreed well with the WCM measurements. Compared to the case with $300 \mathrm{kV}$ of $197 \mathrm{MHz}$ rf cavities used in 2012, the particle leakage rate slowed down due to the reduced bunch intensity at the central bunch area. And the momentum spread of the proton beam was smaller with $100 \mathrm{kV} 197 \mathrm{MHz}$ cavities.

For Fill 17520, there was less particle loss due to the off-momentum momentum aperture limit. During the first hour at store, there was $4.5 \%$ particles moving out of the central bunch area [ $-5 \mathrm{~ns}, 5 \mathrm{~ns}]$. The actual particle loss rate was $5.2 \% / \mathrm{h}$. From both the actual WCM measurements and the simulation, the tail population slightly increased throughout the store.

Figure 19 shows the evolution of bunch intensity from the multiparticle simulation with $300 \mathrm{kV} 197 \mathrm{MHz}$ rf cavities. The red curve shows the bunch intensity only with

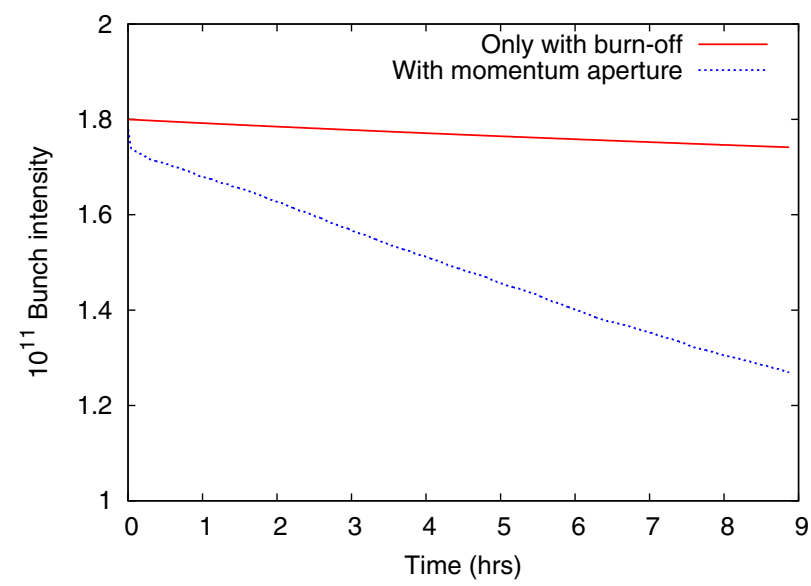

FIG. 19. Evolution of bunch intensity from multiparticle simulation. Momentum aperture $6 \times 10^{-4}$ is applied. Initial beam parameters similar to Fill 16697 are used. the burn-off beam loss. The average particle loss rate at store is $0.32 \% / \mathrm{h}$, which is close to our early analytical estimate.

The blue curve in Fig. 19 shows the evolution of bunch intensity with an off-momentum aperture $6.0 \times 10^{-4}$. The very early particle loss is from particles initially with large off-momentum deviation than the momentum aperture. After the very fast early loss, the particle loss is determined by the IBS growth rate and the momentum aperture. The smaller momentum aperture, the larger particle loss rate is. For an off-momentum aperture $6.0 \times 10^{-4}$, the particle loss rate is $3.5 \% / \mathrm{h}$ from simulation, which is close to the measurements after the first hour at store.

Currently we cannot demonstrate the large fast particle loss in the first hour at store. One reason is that we used a hard momentum aperture in simulation. In RHIC, there is not a momentum collimation system. The real process of particle loss may take a longer time. The second reason is that we could not generate a realistic particle distribution in the bunch tail. Compared to the real bunch profile measurements, there were more particles on both sides of the central $197 \mathrm{MHz}$ rf bucket than the simulation. It would take a relatively longer time for those particles to get lost due to the IBS effect.

In Ref. [29], the longitudinal distribution and particle loss at store with a single frequency of rf system are modeled by numerically solving an integrodifferential diffusion equation. This method was applied to Tevatron at the Fermi National Accelerator Laboratory. As opposed to RHIC, the off-momentum dynamic aperture with beambeam interactions was not the main limiting factor to the beam lifetime at the Tevatron [30-32].

\section{CONCLUSION}

In this article, we presented the operational observations in the past RHIC polarized proton runs. We analyzed the mechanisms for the proton particle loss, transverse emittance growth, and bunch lengthening at store. A strong linear correlation was established between the beam loss rate and the particle leakage rate out of the central bunch area. We concluded that the particle loss was caused by a limited off-momentum dynamic aperture which was reduced by the beam-beam interactions. IBS was identified as the main reason for the growths of transverse emittances and bunch length. It moves particles out of the bunch central area. These particles in the bunch tail with an offmomentum deviation larger than the momentum aperture would get lost. Both analytical calculations and multiparticle simulations largely reproduced the observed evolutions of bunch lengthening and emittance growth over the physics store. Based on these findings, a new store lattice with a much larger off-momentum dynamic aperture was used in the 2015 RHIC $100 \mathrm{GeV}$ proton run. Together with the head-on beam-beam compensation, both the peak and average luminosities were doubled [33]. 


\section{ACKNOWLEDGMENTS}

We would like to thank our colleagues M. Bai, M. Blaskiewicz, A. Drees, H. Huang, M. Minty, C. Montag, V. Ptitsyn, V. Ranjbar, V. Scheoffer, and T. Roser for stimulating discussions during the RHIC polarized proton operation. This work was supported by the US DOE under Contract No. DE-AC02-98CH10886.

\section{APPENDIX: CAUSES OF DYNAMIC APERTURE REDUCTION}

In the following we present the causes of dynamic aperture reduction in the RHIC proton operation.

\section{Chromatic effects with low $\beta^{*} \mathrm{~s}$}

To increase the luminosity, the $\beta^{*} \mathrm{~s}$ at the collisional IPs were reduced. A low $\beta^{*}$ lattice increases the nonlinear chromaticities and reduces the off-momentum dynamic aperture. In the $2009100 \mathrm{GeV}$ run, the $\beta^{*}$ s were further reduced to $0.7 \mathrm{~m}$ from $1.0 \mathrm{~m}$ used in 2008 but shorter intensity and luminosity lifetimes were observed. In the following $100 \mathrm{GeV}$ run in $2012, \beta^{*}$ was increased to $0.85 \mathrm{~m}$ and the beam lifetime was improved.

Figure 20 shows the calculated dynamic apertures with beam-beam interactions versus $\beta^{*}$ s at IP6 and IP8. The particle energy is $100 \mathrm{GeV}$. The tunes without collision are $(28.688,29.685)$. The proton bunch intensity is $1.5 \times 10^{11}$. The transverse emittance is $3.3 \mu \mathrm{m}$. From this plot, the dynamic aperture with beam-beam interactions drops as $\beta^{*}$ decreases. With $\beta^{*}=0.7 \mathrm{~m}$, the calculated dynamic apertures are $4.2 \sigma$ in the Blue ring and $3.6 \sigma$ in the Yellow ring. With a larger $\beta^{*}=0.85 \mathrm{~m}$, they increase to $4.7 \sigma$ and $4.5 \sigma$ respectively.

The main sources to the nonlinear chromaticities are the triplet quadrupoles in the collisional interaction regions (IRs). In these regions, the low $\beta^{*} \mathrm{~s}$ at the IPs lead to a large off-momentum $\beta$-beat $d \beta / d \delta$. To compensate the second

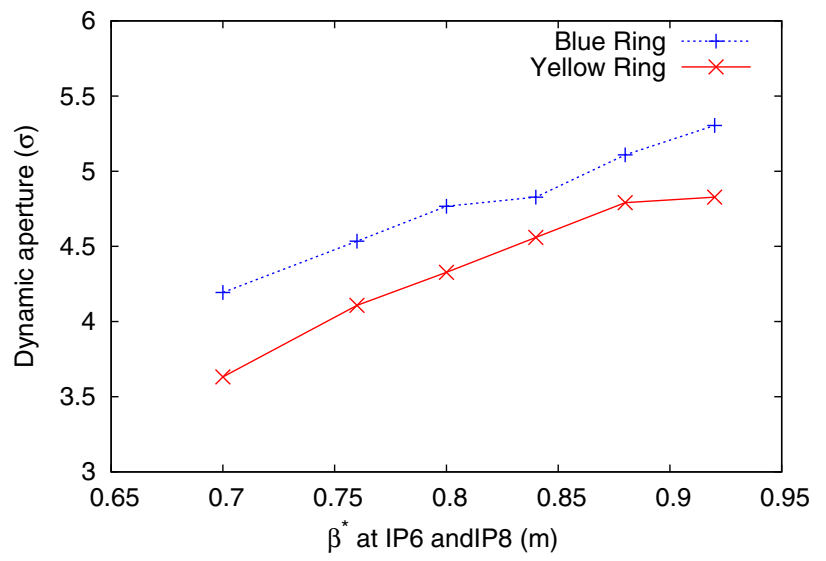

FIG. 20. Calculated dynamic apertures with beam-beam interactions versus the $\beta^{*}$ s at the collisional IP6 and IP8. The particle energy is $100 \mathrm{GeV}$. order chromaticities, we can adjust the betatron phase advances between IP6 and IP8 to be $(k+1 / 2) \pi, k$ is an integer. Another solution is the achromatic-telescopicsqueezing (ATS) scheme which is adopted for the HL-LHC program [34] and was recently implemented in RHIC [35] as a part of the electron lens project.

\section{Third order resonances}

Beam-beam interactions introduce an amplitude-dependent tune shift which is proportional to the bunch intensity. In the limited tune space between $2 / 3$ and $7 / 10$, a large beam-beam parameter generates a large tune spread and pushes the particles in the bunch core toward the third order resonances at $Q_{x, y}=2 / 3$. Even with a low beam-beam parameter in the $2006100 \mathrm{GeV}$ proton run, we already noticed that a working point above the diagonal gave a worse beam-beam lifetime than the one below diagonal. Both simulation and experiments show that the stop-band of the horizontal third order resonance is wider than that of the vertical third order resonance.

Figure 21 shows the calculated dynamic aperture with beam-beam interactions in a tune scan along the diagonal in the tune space between $2 / 3$ and $7 / 10$. The $\beta^{*}$ s at IP6 and IP8 are $1.0 \mathrm{~m}$, which is the same as in 2006 . The bunch intensity is $1.5 \times 10^{11}$ and the transverse rms emittance $3.3 \mu \mathrm{m}$. The initial relative momentum deviation is $12.5 \times 10^{-4}$. The difference between the fractional horizontal and vertical tunes is kept at 0.005 . The horizontal axis is the lower tune value, which can be the horizontal or the vertical tune depending whether the working point is above or below the diagonal.

We can see on Fig. 21 that the dynamic aperture drops when the working point moves toward the third order resonances. At the same distance from the third order resonances, a working point below the diagonal gives a larger dynamic aperture than one above the diagonal.

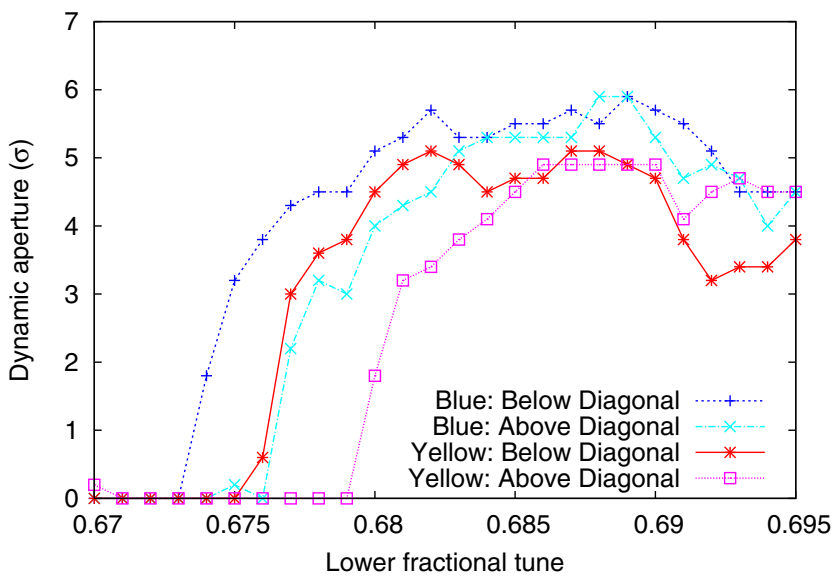

FIG. 21. Calculated dynamic aperture in tune scans above and below the diagonal line. The horizontal tune is the lower lattice tune. 
The Blue ring has a larger dynamic aperture than the Yellow ring. The reason is that the negative second order chromaticities in the Yellow ring pushes the tunes of offmomentum particles closer to the third order resonances than for the Blue ring.

The main sources to the third order resonance driving terms are located in the collisional IRs. In RHIC, we use the local IR normal and skew sextupole correctors to correct the third order resonances. Without collision, by pushing the tunes close to the third order resonances, we could reduce the beam loss by adjusting the local IR sextupole correctors. However, when bringing the beams into collision, the effect of these corrections were not conclusive. Possible explanation is that the beam lifetime with collisions is mainly determined by beam-beam interactions, even though the third order resonance correction could possibly yield some additional tune space.

\section{Limited tune space}

With a larger bunch intensity in the $2013255 \mathrm{GeV}$ proton run, to maintain the proton polarization, the beambeam tune footprint had to be placed closer to the third order resonance. Operational experience shows that a vertical tune higher than 0.686 reduces the polarization lifetime. In addition setting the tune close to $7 / 10$ will introduce large beam losses. Since the beam-beam tune footprint was close to the third order resonances, we normally observed mild transverse emittance blow-up right after collision during routine physics stores in 2013.

Figure 22 shows the peak luminosity and the luminosityderived emittances when we pushed the proton bunch intensity during the $2013255 \mathrm{GeV}$ proton run. Each point in the plot represents one store. The horizontal axis is the average bunch intensity of the Blue and Yellow rings. These data are taken at 5 min after physics data taking was declared. On this plot we can see that when the bunch

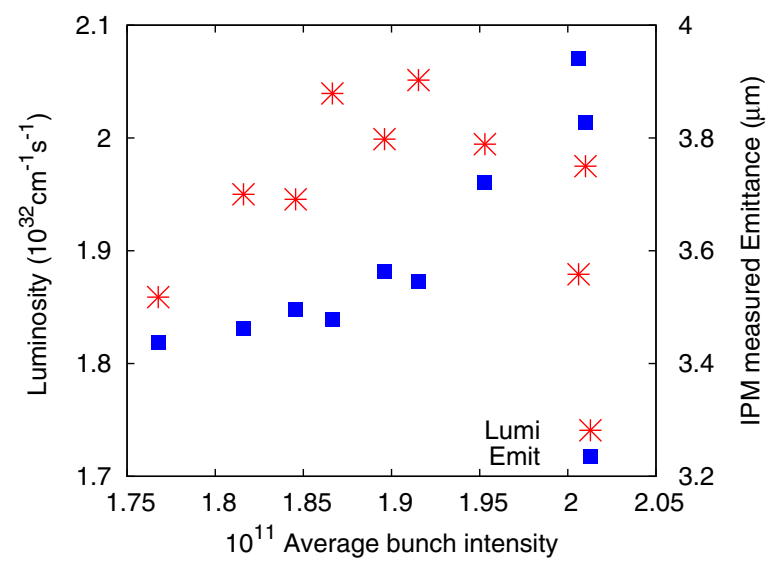

FIG. 22. Luminosities and the luminosity-derived emittances in the $2013255 \mathrm{GeV}$ proton run when we pushed the proton bunch intensities beyond $2.0 \times 10^{11}$. intensity was larger than $1.9 \times 10^{11}$, the transverse emittance was blown up and the peak luminosity was reduced.

To reduce the head-on beam-beam tune spread, two electron lenses have been installed in RHIC [36], one in each ring. Partial head-on beam-beam compensation is used to avoid overcompensation. To cancel the nonlinear head-on beam-beam resonances, the betatron phase advances between IP8 and the center of electron lenses is set to $k \pi$. Electron lenses were also installed in the Tevatron for long-range beam-beam compensation and other purposes [37-41].

[1] M. A. Furman and M. S. Zisman, in Handbook of Accelerator Physics and Engineering, edited by A. Chao, K. H. Mess, M. Tigner, and F. Zimmermann (World Scientific, Singapore, 2013), Ch. 4, p. 311.

[2] W. Fischer, RHIC Run Overview, website: http://www .rhichome.bnl.gov/RHIC/Runs/.

[3] T. Sen, B. Erdelyi, M. Xiao, and V. Boocha, Phys. Rev. Accel. Beams 7, 041001 (2004).

[4] K. Hirata, in Handbook of Accelerator Physics and Engineering, edited by A. Chao, K. H. Mess, M. Tigner, and F. Zimmermann (World Scientific, Singapore, 2013), Chap. 2, p. 169.

[5] H. Kim, T. Sen, N. P. Abreu, and W. Fischer, Phys. Rev. Accel. Beams 12, 031001 (2009).

[6] R. Calaga, W. Fischer, G. Robert-Demolaize, and N. Milas, Phys. Rev. Accel. Beams 14, 091001 (2011).

[7] Y. Luo, P. Cameron, A. Dellapenna, L. Hoff, A. Marusic, S. Peggs, C. Schultheiss, and R. Jones, Phys. Rev. Accel. Beams 9, 124001 (2006).

[8] P. Cameron, A. DellaPenna, L. Hoff, Y. Luo, A. Marusic, C. Schultheiss, S. Tepikian, M. Gasior, R. Jones, and C. Y. Tan, Phys. Rev. Accel. Beams 9, 122801 (2006).

[9] R. Michnoff et al., in Proceedings of the 24th Particle Accelerator Conference, PAC-2011, New York, 2011 (IEEE, New York, 2011), p. 41.

[10] M. Minty et al., in Proceedings of the 24th Particle Accelerator Conference, PAC-2011, New York, 2011 (IEEE, New York, 2011).

[11] W. Fischer, J. M. Brennan, M. Blaskiewicz, and T. Satogata, Phys. Rev. Accel. Beams 5, 124401 (2002).

[12] W. Fischer et al., Phys. Rev. Accel. Beams 11, 041002 (2008).

[13] S. Y. Zhang, H. C. Hseuh, P. Thieberger, and D. Trbojevic, Phys. Rev. Accel. Beams 8, 123201 (2005).

[14] S. Y. Zhang and V. Ptitsyn, Phys. Rev. Accel. Beams 11, 051001 (2008).

[15] Y. Luo, in Proceedings of the International Particle Accelerator Conference, Kyoto, Japan (ICR, Kyoto, 2010).

[16] Y. Luo, in Proceedings of the 2015 International Particle Accelerator Conference, Richmond, VA, USA (JACow, Richmond, 2015).

[17] M. J. Rhoades-Brown and M. Harrison, BNL Report No. 47070, 1991 (unpublished). 
[18] J. D. Jackson, Classic Electrodynamics (John Wiley- Sons, New York, 1975).

[19] B. Frankzke, CERN Yellow Report No. 92-01, 1992, pp. 100120.

[20] V. Lebedev, in Handbook of Accelerator Physics and Engineering, edited by A. Chao, K. H. Mess, M. Tigner, and F. Zimmermann (World Scientific, Singapore, 2013), Chap. 2, p. 155.

[21] C. Bernardini, G. F. Corazza, G. Di Giugno, G. Ghigo, J. Haissinski, P. Marin, R. Querzoli, and B. Touschek, Phys. Rev. Lett. 10, 407 (1963).

[22] A. Piwinski, Report No. DESY 98-179, 1998 (unpublished).

[23] A. Piwinski, in Frontiers of Particle Beams, edited by M. Month and S. Turner (Springer, New York, 1988), p. 297.

[24] J. Bjorken and S. Mtingwa, Part. Accel. 13, 115 (1983).

[25] M. Martini, Part. Accel. 17, 1 (1985).

[26] Y. Luo, W. Fischer, M. Blaskiewicz, J. M. Brennan, N. Kling, K. Mernick, and T. Roser, Phys. Rev. Accel. Beams 17, 081003 (2014).

[27] A. Fedotov, BNL Report No. RHIC/AP/168, 2009 (unpublished).

[28] M. Blaskiewicz, J. M. Brennan, and F. Severino, Phys. Rev. Lett. 100, 174802 (2008).

[29] V. Lebedev, in Proceedings of the 20th ICFA Advanced Beam Dynamics Workshop on High Intensity and High Brightness Hadron Beams, Batavia, IL, USA (JACow, Batavia, 2004).
[30] V. Shilsev, Y. Alexahin, V. Lebedev, P. Lebrun, R. Moore, T. Sen, A. Tollestrup, A. Valishev, and X. Zhang, Phys. Rev. Accel. Beams 8, 101001 (2005).

[31] V. Lebedev, in Proceedings of the 20th Particle Accelerator Conference, PAC-2003, Portland, OR, 2003 (IEEE, New York, 2003).

[32] R. Carrigan et al., in Accelerator Physics at the Tevatron Collider, edited by V. Lebedev and V. Shiltsev (Springer, New York, 2014), Chap. 6.

[33] W. Fischer et al., Phys. Rev. Lett. 115, 264801 (2015).

[34] S. Fartoukh, Phys. Rev. Accel. Beams 16, 111002 (2013).

[35] S. White et al., Report No. BNL RHIC/AP/519, 2014 (unpublished).

[36] Y. Luo, Phys. Rev. Accel. Beams 15, 051004 (2012).

[37] V. Shiltsev, V. Danilov, D. Finley, and A. Sery, Phys. Rev. Accel. Beams 2, 071001 (1999).

[38] V. Shiltsev, Y. Alexahin, K. Bishofberger, V. Kamerdzhiev, G. Kuznetsov, and X.-L. Zhang, Phys. Rev. Lett. 99, 244801 (2007).

[39] V. Shiltsev et al., Phys. Rev. Accel. Beams 11, 103501 (2008).

[40] X. Zhang, K. Bishofberger, V. Kamerdzhiev, V. Lebedev, V. Shiltsev, R. Thurman-Keup, and A. Tollestrup, Phys. Rev. Accel. Beams 11, 051002 (2008).

[41] G. Stancari, in Proceedings of the 25th Particle Accelerator Conference, PAC-2013, Pasadena, CA, 2013 (IEEE, New York, 2013). 